\title{
The risk of infective endocarditis among people who inject drugs: a retrospective, population- based time series analysis
}

\author{
Matthew A. Weir MD MSc, Justin Slater MSc, Racquel Jandoc MSc, Sharon Koivu MD, Amit X. Garg MD PhD, \\ Michael Silverman MD
}

Cite as: CMAJ 2019 January 28;191:E93-9. doi: 10.1503/cmaj.180694

\begin{abstract}
BACKGROUND: Infective endocarditis is an increasingly common complication among people who inject drugs. We conducted this study to determine whether the removal of traditional controlled-release oxycodone from the Canadian market would be associated with an increase in the use of hydromorphone and an increased risk of infective endocarditis.
\end{abstract}

METHODS: We conducted a retrospective, population-based time series analysis using the linked health administrative databases of Ontario, Canada. We measured the quarterly risk of admissions for infective endocarditis related to injection drug use and changes in opioid prescription rates from 2006 to 2015 . We set the intervention point at the fourth quarter of 2011, when traditional controlledrelease oxycodone was removed from the Canadian market.

RESULTS: We observed an increase in the risk of admissions for infective endocarditis related to injection drug use during the study period. Before the intervention point, we observed a mean of 13.4 admissions per quarter, and after the intervention, we observed a mean of 35.1 admissions per quarter. However, no significant change in this risk occurred at the intervention point. Rather, the risk of infectious endocarditis appeared to have increased earlier and in parallel with the rise in hydromorphone prescriptions. Hydromorphone represented $16 \%$ of all opioid prescriptions at the start of the observation period and $53 \%$ by the end.

INTERPRETATION: The risk of infective endocarditis related to injection drug use is increasing and is temporally associated with increasing prescriptions for hydromorphone. This relation warrants further exploration.
$\mathbf{T}$ he illicit use of prescription opioids is increasing in North America. ${ }^{1,2}$ Oral opioids diverted for illicit use are often crushed and dissolved for intravenous injection. In addition to the risks of overdose and the transmission of blood-borne viruses, this method of opioid use confers a substantial risk of infective endocarditis through breaks in sterile technique and injection of excipients. ${ }^{3,4}$

At our institution, we noticed an increase in cases of infective endocarditis related to injection drug use, a trend that has been documented in Australia, ${ }^{5}$ the United States ${ }^{6}$ and Sweden. ${ }^{7}$ Through existing literature, ${ }^{8}$ and reports from field workers, we learned of differences in injection practices between 2 commonly injected opioids, hydromorphone and controlled-release oxycodone, that could result in different risks of contamination and bacteremia. When hydromorphone is injected, the injection equipment is frequently reused and shared with others because residual drug is believed to remain in the equipment, but these contaminationprone practices are not observed when people inject traditional controlled-release oxycodone. ${ }^{8}$ Therefore, we suspected that an increase in hydromorphone use could be associated with an increase in infective endocarditis related to injection drug use.

The impetus for increased hydromorphone use occurred when a tamper-resistant formulation of controlled-release oxycodone (OxyNEO, Purdue Pharma) replaced the traditional formulation of this drug (OxyContin, Purdue Pharma) in the fourth quarter of 2011. We sought to test our hypothesis that the removal of traditional controlled-release oxycodone from the Canadian market would be associated with an increase in the use of hydromorphone and an increased risk of infective endocarditis by analyzing routinely collected data. 


\section{Methods}

\section{Study design}

We conducted a retrospective population-based time series analysis between Apr. 1, 2006, and Sept. 30, 2015, using the linked health administrative databases of Ontario, Canada. Citizens of Ontario have universal access to physician services and inpatient care. Prescription drug coverage is provided to those aged 65 years and older, and to those who meet needs-based criteria (e.g., people receiving home care services or provincial social assistance, people enrolled in a provincial disability support program, and people with a high ratio of drug costs to income). The reporting of this study follows guidance for reporting observational studies (Supplemental Table 1, Appendix 1, available at www.cmaj.ca/lookup/suppl/doi:10.1503/cmaj.180694/-/DC1). ${ }^{9}$

\section{Data sources}

We used 5 linked databases to conduct this study. We gathered information on hospital and emergency department admissions using the Canadian Institute for Health Information's Discharge Abstract Database and the National Ambulatory Care Reporting System database. For information on physician services, we used the Ontario Health Insurance Plan database, and for demographic data and vital statistics, we used the Registered Persons Database. We used the Ontario Drug Benefit database to ascertain prescription rates (Drug Identification Numbers obtained from the IMS Brogan Drug Information Database). These data sets were linked using encoded identifiers and analyzed at ICES. The authors (M.A.W., J.S. and A.X.G.) had access to the databases. Programming of data extraction was carried out by J.S. according to a prespecified data creation plan that is available on request.

\section{Study population}

To study trends in infective endocarditis related to injection drug use, we first estimated the size of the population of people who inject drugs each quarter between the second quarter of 2006 and third quarter of 2015. We accomplished this using a set of administrative codes and characteristics that we previously designed and validated to identify people who inject drugs (Supplemental Table 2, Appendix 1). ${ }^{10}$ This definition includes patients aged 18 to 55 years who had hospital admissions in the preceding 6 months with diagnoses of opioid, stimulant or combined drug abuse, or infection with hepatitis $C$. The age restriction reflects the distribution of ages in previously described cohorts of people who inject drugs and in our own validation study. ${ }^{7,11,12}$ Compared with chart review, this definition has been shown to have a sensitivity of $93 \%$ and a positive predictive value of $83 \% .^{10}$

We defined infective endocarditis using administrative codes (Supplemental Table 3, Appendix 1). We defined admissions with infective endocarditis as being related to injection drug use if an admission occurred within 6 months of an admission that qualified the patient as a person who injects drugs. Admissions with infective endocarditis occurring within 30 days of a previous admission with infective endocarditis were considered to be relapses and were excluded. To identify trends over time, we measured quarterly rates of infective endocarditis related to injection drug use.

\section{Intervention}

We hypothesized that the loss of traditional controlled-release oxycodone from the Canadian market in the fourth quarter of 2011 would be temporally associated with an increase in the use of hydromorphone and an increase in the risk of infective endocarditis related to injection drug use. To explore this, we looked for changes in the quarterly risk of injection drug use-related infective endocarditis, using the fourth quarter of 2011 as an intervention point, and we looked for changes in trends in the use of controlled-release oxycodone and hydromorphone.

\section{Statistical analysis}

We compared the prevalence of baseline characteristics between those with and without injection drug use-related infective endocarditis using standardized differences, which describe a difference between group means as a percentage of the pooled standard deviation. Standardized differences greater than $10 \%$ represent meaningful imbalances. ${ }^{13,14}$ We used change-point regression to determine whether there was a difference in the rate of infective endocarditis related to injection drug use before and after the fourth quarter of 2011. Data for 23 quarters before the intervention and 15 quarters after the intervention were available for analysis. The increase in the rate of infective endocarditis related to injection drug use was estimated using linear regression. We used an F-test to determine whether there was a significant difference in the slope before and after the intervention point. ${ }^{15}$ Our model met the assumptions for a linear model, and the Durbin-Watson test for autocorrelation was not significant. ${ }^{16,17}$ We considered results significant at the 0.05 level using 2-tailed tests. We conducted all analyses with SAS 9.4.

\section{Ethics approval}

This study was approved by the institutional review board at the Sunnybrook Health Sciences Centre, Toronto.

\section{Results}

During the study period (second quarter of 2006 to the third quarter of 2015), we observed a total of 60529 admissions of people who inject drugs (a flow diagram depicting the cohort construction is shown in Supplemental Figure S1, Appendix 1). Of these, 733 admissions had evidence of infective endocarditis related to injection drug use. Compared with those without infective endocarditis, patients admitted with infective endocarditis related to injection drug use were younger, were more likely to be male and generally had a higher burden of comorbidities, including infection with HIV and hepatitis C (Table 1).

\section{Trends in infective endocarditis related to injection drug use}

Admission rates for people who inject drugs remained stable during the study period, with a mean of 1622 admissions per quarter (range 1381-1867 admissions per quarter). However, as shown in Figure 1, the risk of infective endocarditis among people who inject drugs increased during the study period. Before the intervention point (fourth quarter of 2011), we observed a mean of 13.4 admissions per quarter, and after the 
intervention point, we observed a mean of 35.1 admissions per quarter. However, at the intervention point, we found no significant immediate change in the risk of infective endocarditis ( $p=0.4$ ), and the slope after the intervention was not significantly different from the pre-intervention slope $(p=0.8)$. Rather, Figure 1 suggests that the increase in risk predated the removal of controlled-release oxycodone from the Canadian market.
Correlation with increasing use of hydromorphone

For each quarter of the study period, we identified all prescriptions for fentanyl, morphine, meperidine, oxycodone and hydromorphone. We observed a mean of 143606 unique opioid prescriptions per quarter. Whereas this rate remained stable during the study period, the proportion of opioid prescriptions for controlled-release oxycodone and hydromorphone changed

Table 1 (part 1 of 2): Characteristics of patients with evidence of injection drug use, comparing those with and without infective endocarditis

\section{No. $(\%)$ of patients*}

Injection drug use without infective endocarditis

Characteristic $n=59796$
Injection drug use with infective endocarditis $n=733$
Standardized difference, $\uparrow \%$

\section{Demographic data}

Age at cohort entry, yr

Mean $\pm S D, y r$

Median (IQR), yr

18-25

26-35

36-45

46-55

Sex, female

Residential status

Urban

Rural

Missing

Socioeconomic status

1 (lowest)

2

3

4

5 (highest)

\section{Comorbidities}

Alcohol abuse

Bipolar disorder

Chronic liver disease

Depression and/or anxiety

Hepatitis B

Hepatitis C

HIV

Schizophrenia

Self-harm

\section{Medication use}

Antibiotics

Anticoagulants

Antipsychotics

Benzodiazepines

Lithium

Opioids
$40.18 \pm 10.79$

42 (31-50)

7701 (12.9)

13014 (21.8)

14892 (24.9)

24189 (40.5)

26324 (44.0)

51756 (86.6)

8011 (13.4)

$29(0.0)$

23187 (38.8)

12876 (21.5)

10365 (17.3)

7531 (12.6)

5837 (9.8)

3658 (6.1)

733 (1.2)

$4220(7.1)$

11770 (19.7)

$165(0.3)$

8609 (14.4)

2209 (3.7)

1065 (1.8)

8244 (13.8)

14544 (24.3)

$590(1.0)$

9239 (15.5)

12909 (21.6)

$613(1.0)$

23006 (38.5)
$36.33 \pm 9.45$

$36(28-44) \quad 42$

$99(13.5) \quad 2$

$256(34.9) \quad 30$

$230(31.4) \quad 14$

$148(20.2)$

$390(53.2) \quad 18$

$640(87.3) \quad 2$

$93(12.7) \quad 2$

$0(0.0) \quad 3$

$320(43.7) \quad 10$

$162(22.1)$

$113(15.4) \quad 5$

$77(10.5) \quad 7$

$61(8.3) \quad 5$

$15(2.0) \quad 21$

$<6 \ddagger \quad 7$

$22(3.0) \quad 19$

$120(16.4)$

$0(0.0) \quad 7$

$135(18.4) \quad 11$

$46(6.3) \quad 12$

$<6 \ddagger \quad 10$

$94(12.8) \quad 3$

$223(30.4) \quad 14$

$15(2.0)$

$94(12.8) \quad 8$

$144(19.6) \quad 5$

$<6 \ddagger \quad 7$

$366(49.9) \quad 23$ 
Table 1 (part 2 of 2): Characteristics of patients with evidence of injection drug use, comparing those with and without infective endocarditis

No. (\%) of patients*

Characteristic

Injection drug use without infective endocarditis

$n=59796$
Injection drug use with infective

endocarditis

$n=733$
Standardized difference, $\uparrow \%$

General measures of comorbidity

No. of unique drug prescriptions filled in 1 year

$\begin{array}{lc}\text { Mean } \pm \text { SD } & 4.08 \pm 5.59 \\ \text { Median (IQR) } & 1(0-7)\end{array}$

$1.17 \pm 2.29$

$0(0-1)$

Median (IQR)

No. of emergency department visits in 1 year

Mean \pm SD

Median (IQR)

$4.58 \pm 8.43$

$2(1-5)$

No. of family physician visits in 1 year

$\begin{array}{lc}\text { Mean } \pm \text { SD } & 21.05 \pm 25.07 \\ \text { Median (IQR) } & 11(5-27)\end{array}$

$\begin{array}{cc}3.85 \pm 4.80 & 4 \\ 2(0-6) & 7 \\ 1.10 \pm 1.95 & 3 \\ 0(0-1) & 1\end{array}$

$5.01 \pm 6.43$

$3(1-7)$

$22.78 \pm 24.76$

$13(5-33)$
29

4

7

3

1

6

7

7

Note: $\mathrm{HIV}=$ human immunodeficiency virus, $\mathrm{IQR}=$ interquartile range, $\mathrm{SD}=$ standard deviation

*Unless stated otherwise.

†The standardized difference is the difference between group means expressed as a percentage of the pooled standard deviation. Standardized differences greater than $10 \%$ represent meaningful imbalances.

fIn accordance with ICES privacy policies, cell sizes $\leq 5$ cannot be reported, and are instead expressed here as $<6$.

markedly. As shown in Figure 2, the percentage of opioid prescriptions attributed to traditional controlled-release oxycodone decreased quickly at the time of its removal from the market, and the percentage attributed to hydromorphone increased steadily from $16 \%$ at the start of the study period to $53 \%$ by the end. Figure 3 shows a parallel increase in hydromorphone's percentage of all opioid prescriptions and the risk of admission with infective endocarditis related to injection drug use.

\section{Interpretation}

In this study, we identified a substantial and progressive increase in the risk of infective endocarditis among people who inject drugs, beginning in 2010. This increase in overall risk ${ }^{5-7}$ and the higher risk among men ${ }^{18}$ is consistent with findings from other jurisdictions. We expected this increase to occur in the fourth quarter of 2011, when traditional controlled-release oxycodone was removed from the Canadian market; however, the increase in risk predated this event. Although our observations do not support our hypothesis that the loss of controlled-release oxycodone increased the use of hydromorphone, they do support our suspicion that hydromorphone may be playing a role in the increasing risk of infective endocarditis.

We formulated this hypothesis because injection equipment used to prepare hydromorphone may be more likely to be contaminated with bacteria than equipment used to prepare traditional controlled-release oxycodone. To understand the reasoning behind this hypothesis, knowledge of how oral formulations of opioids are prepared for intravenous injection is required. Although techniques vary, injecting oral formulations of opioids often involves crushing the drug and putting it into solution with a combination of acid, water and heat. These steps often take place in a "cooker," a small metal vessel that is either homemade or supplied (along with ascorbic acid, sterile water, filters and syringes) by some public health units in Ontario. Although these steps are common to both traditional controlledrelease oxycodone and hydromorphone, the poor solubility of hydromorphone is what may lead to reuse and contamination of equipment. The slow release of oxycodone from tablets of controlled-release oxycodone is achieved through the combination of 2 retardants, a water-insoluble polymer and a wax. ${ }^{19}$ Using a cold water extraction method, or through cooling following a heated extraction method, the water-soluble drug can be easily separated from the insoluble retardants. ${ }^{20}$ However, controlled-release hydromorphone consists of polymer-coated beads that are difficult to crush and produce a turbid solution. ${ }^{8}$ Solids that fail to go into solution remain in the cookers and filters, and are believed to contain residual hydromorphone. ${ }^{8}$ As documented by Roy and colleagues, this leads to storage, reuse and resale of cookers and filters used to prepare hydromorphone, ${ }^{8}$ which may lead to an increased risk of contamination and subsequent bacteremia.

The strengths of our study relate to 2 features of Ontario's health administrative databases: their size and inclusivity. The size 


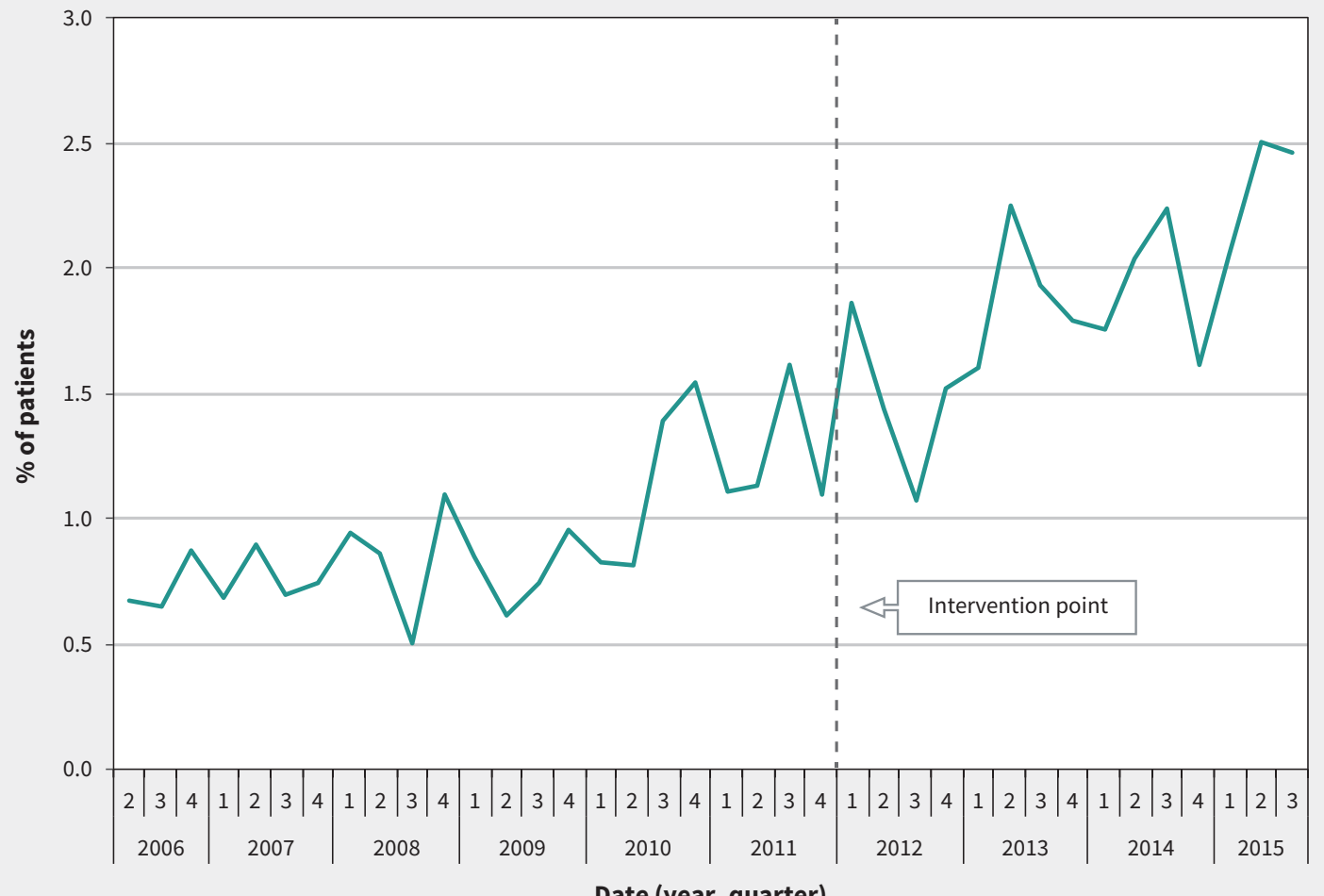

Date (year, quarter)

Figure 1: Quarterly percentage of patients with evidence of injection drug use admitted with infective endocarditis. The point at which traditional controlled-release oxycodone was removed from the market is marked as "intervention point."

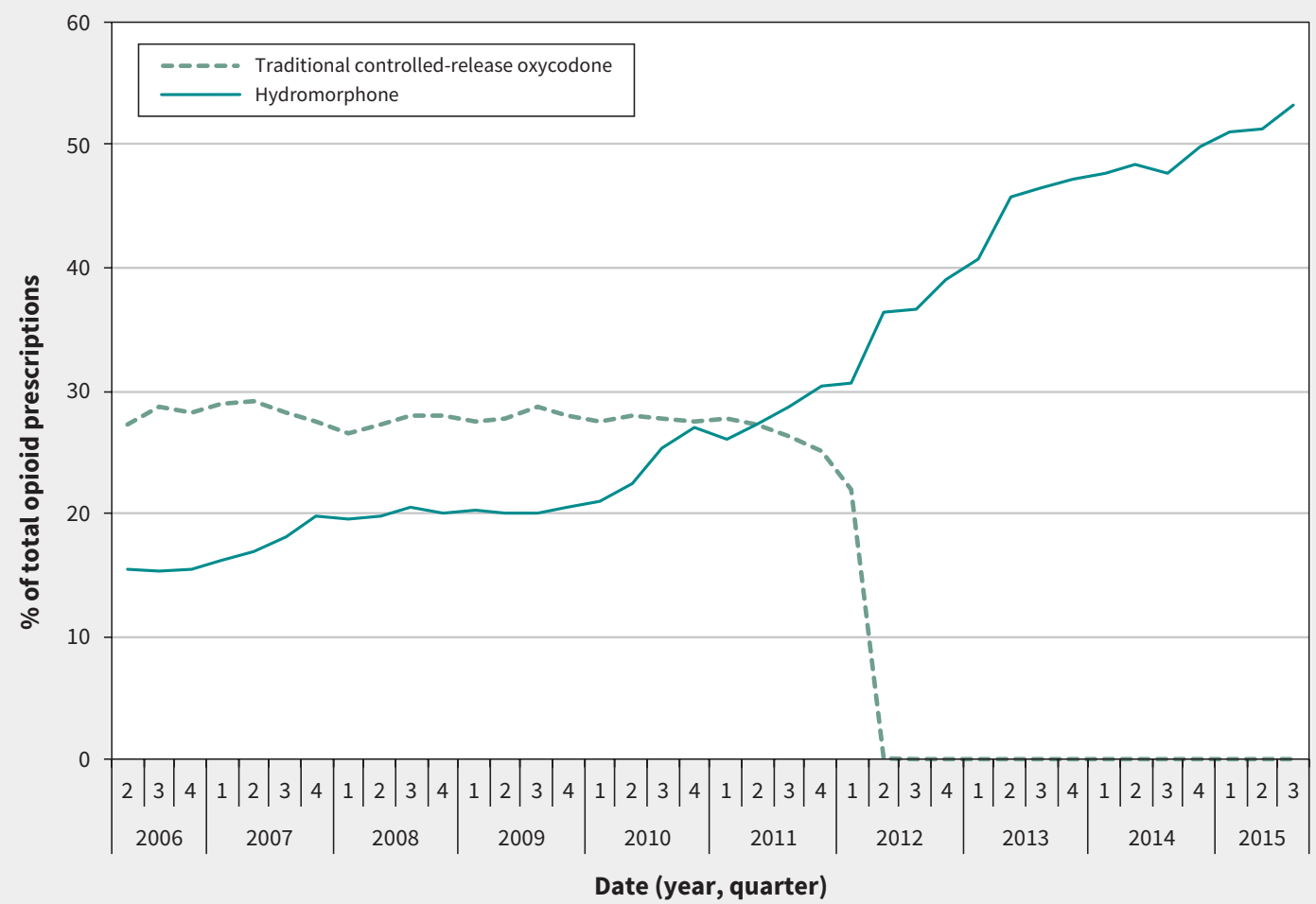

Figure 2: Quarterly prescriptions for traditional controlled-release oxycodone and hydromorphone as percentages of total opioid prescriptions. 


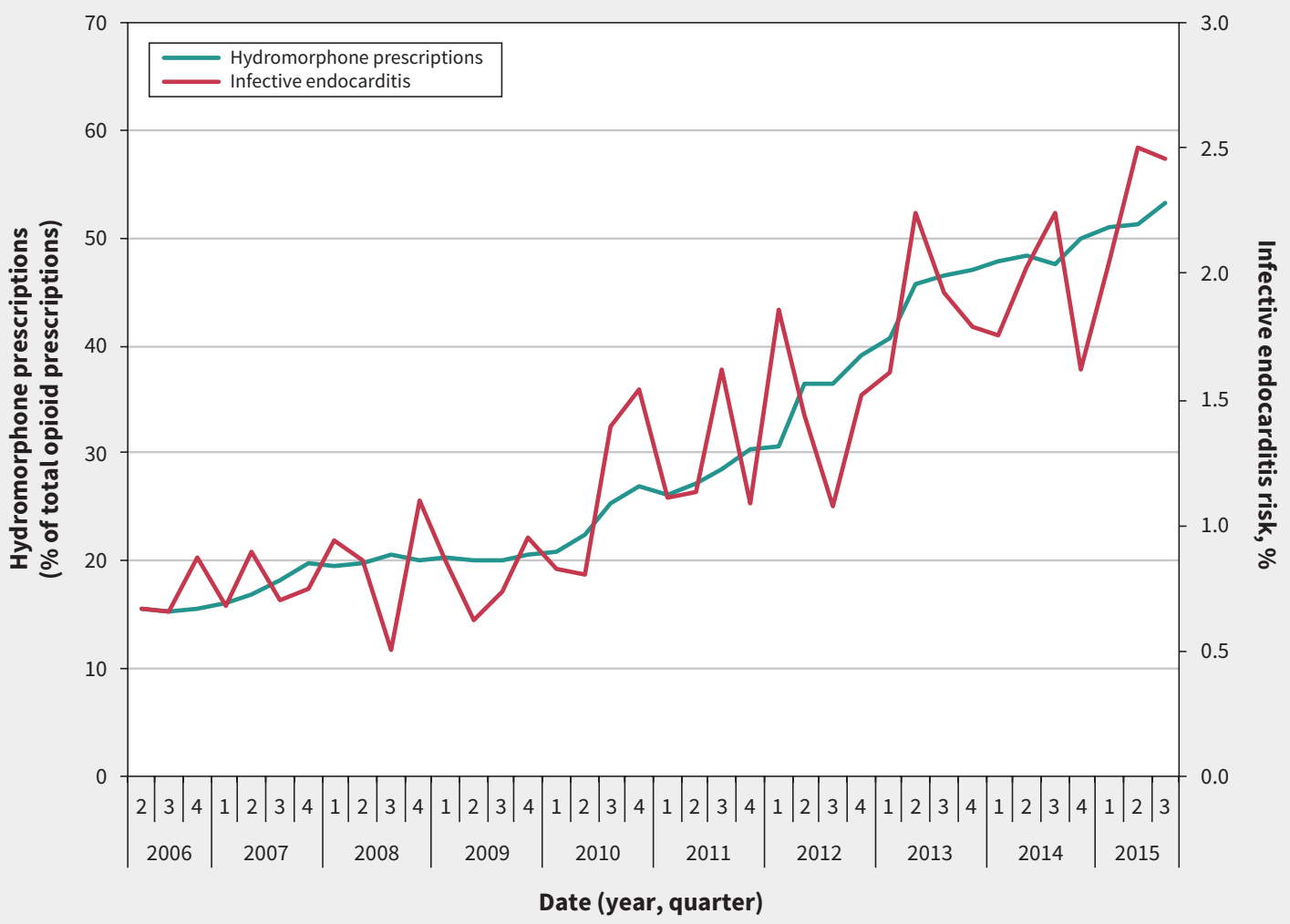

Figure 3: Quarterly prescriptions for hydromorphone as a percentage of total opioid prescriptions and the risk of infective endocarditis related to injection drug use.

of these databases allowed us to produce meaningful estimates of risk for a relatively rare outcome. Compared with databases with demographic or financial limitations to enrolment, Ontario's universal health coverage allowed us to collect better data on a marginalized and economically disadvantaged population.

\section{Limitations}

When interpreting our study's findings, it is important to consider the limitations of our population definition, our exposure definition and the plausibility of alternative explanations for the observed increase in the risk of infective endocarditis. Our definitions of people who inject drugs and of infective endocarditis were limited by our use of administrative data. We underestimated the number of people who inject drugs because we were able to count only those who presented to hospital, and the administrative definition of infective endocarditis has only a moderate positive predictive value of $78 \% .{ }^{21}$ However, we used these definitions consistently over the study period, and their limitations applied equally to periods of low and high risk. Therefore, the increasing risk of infective endocarditis related to injection drug use we identified is relatively unaffected by the operating characteristics of these definitions.

Our exposure definitions were also subject to the limitations of administrative data. Although opioid prescription rates have been shown to correlate well with the total number of opioid deaths and opioid treatment admissions, ${ }^{22,23}$ there are 2 features of these data that could weaken the association between "prescriptions filled" and "drugs injected." First, the Ontario Drug Benefit database captures information on prescriptions filled by people older than 65 years or by people who fulfill needs-based criteria (see Methods); these criteria may have made our estimates of opioid use less representative of opioid use across the entire province. This limitation could be more pronounced in our study because of the age restriction of 55 years and younger. Second, our drug use data are limited to prescription pharmaceuticals. Although fentanyl prescriptions were rare (less than $2 \%$ of total prescriptions) and did not change over the course of the study, fentanyl-related overdoses have increased substantially since $2013 .{ }^{24}$ This discrepancy is likely the result of fentanyl manufactured and sold outside of the pharmaceutical industry. Similar to heroin or other black market opioids, we cannot assess the use of this kind of drug, nor can we rule out its role in the observed increase in infectious endocarditis related to injection drug use.

Although we have proposed that increasing hydromorphone exposure is a possible cause of the increasing risk of infective endocarditis, other factors may have influenced this trend. The likelihood of diagnosing infective endocarditis may have increased over time if physicians noticed more cases and adopted a higher index of suspicion. A loss of resources or changes in policy regarding harm-reduction measures in Ontario could have influenced our findings, but these services have either remained static or expanded during the study period. A guideline suggesting less frequent use of endocarditis prophylaxis was published in $2007,{ }^{25}$ but this is not likely the cause of 
the increased risk we observed beginning in 2010. OxyNEO was introduced to the Canadian market near the point at which the risk of infective endocarditis started to increase; however, despite this temporal association, we found very low prescription rates for this drug, likely owing to the restrictions placed on its use by the Ontario Public Drug Programs. Our study's most important limitation, however, is its inability to establish a causal relation between hydromorphone use and infective endocarditis.

\section{Conclusion}

We observed a substantial increase in the risk of infective endocarditis among people who inject drugs, which is associated with hydromorphone's increasing share of the prescription opioid market. Both the rise in this severe complication of injection drug use and the possible association with hydromorphone require further study. Although our data show a compelling temporal association between hydromorphone use and infective endocarditis, further research is required to make a case for causation. This work should endeavour to draw closer links between exposure and outcome, using individual prescription drug exposure, urine toxicology results or sampling of used drug injection equipment.

\section{References}

1. Rudd RA, Aleshire N, Zibbell JE, et al. Increases in drug and opioid overdose deaths - United States, 2000-2014. MMWR Morb Mortal Wkly Rep 2016;64:1378-82.

2. Fischer B, Gooch J, Goldman B, et al. Non-medical prescription opioid use, prescription opioid-related harms and public health in Canada: an update 5 years later. Can J Public Health 2014;105:e146-9.

3. Moss R, Munt B. Injection drug use and right sided endocarditis. Heart 2003; 89:577-81.

4. Olubamwo O, Onyeka IN, Aregbesola A, et al. Association between route of illicit drug administration and hospitalizations for infective endocarditis. SAGE Open Med 2017;5:1-5.

5. Tung MKY, Light M, Giri R, et al. Evolving epidemiology of injecting drug useassociated infective endocarditis: a regional centre experience. Drug Alcohol Rev 2015;34:412-7.

6. Cooper HLF, Brady JE, Ciccarone D, et al. Nationwide increase in the number of hospitalizations for illicit injection drug use-related infective endocarditis. Clin Infect Dis 2007;45:1200-3.

7. Asgeirsson $\mathrm{H}$, Thalme A, Weiland $\mathrm{O}$. Low mortality but increasing incidence of Staphylococcus aureus endocarditis in people who inject drugs: experience from a Swedish referral hospital. Medicine (Baltimore) 2016;95:e5617.
8. Roy E, Arruda N, Bourgois P. The growing popularity of prescription opioid injection in downtown Montréal: new challenges for harm reduction. Subst Use Misuse 2011;46:1142-50.

9. Nicholls SG, Quach P, von Elm E, et al. The REporting of studies Conducted using Observational Routinely-collected health data (RECORD) statement: methods for arriving at consensus and developing reporting guidelines. PLOS One 2015;10:e0125620.

10. Ball LJ, Sherazi A, Laczko D, et al. Validation of an algorithm to identify infective endocarditis in people who inject drugs. Med Care 2018;56:e70-5.

11. Wurcel AG, Anderson JE, Chui KKH, et al. Increasing infectious endocarditis admissions among young people who inject drugs. Open Forum Infect Dis 2016;3:ofw157.

12. Hartman L, Barnes E, Bachmann L, et al. Opiate injection-associated infective endocarditis in the southeastern United States. Am J Med Sci 2016;352:603-8.

13. Austin PC. Using the standardized difference to compare the prevalence of a binary variable between two groups in observational research. Commun Stat Simul Comput 2009;38:1228-34.

14. Mamdani M, Sykora K, Li P, et al. Reader's guide to critical appraisal of cohort studies: 2. Assessing potential for confounding. BMJ 2005;330:960-2.

15. Julious SA. Inference and estimation in a changepoint regression problem. Statistician 2001;50:51-61 doi: 10.1111/1467-9884.00260.

16. Kleinbaum D, Kupper DG, Nizam A, et al. Applied regression analysis and other multivariable methods. 4th ed. Pacific Grove (CA): Duxbury Press; 2007.

17. Brocklebank JC, Dickey DA. SAS for forecasting time series. 2nd ed. Cary (NC): SAS Institute Inc.; 2003.

18. Wright $\mathrm{A}$, Otome $\mathrm{O}$, Harvey $\mathrm{C}$, et al. The current epidemiology of injecting drug use-associated infective endocarditis in Victoria, Australia in the midst of increasing crystal methamphetamine use. Heart Lung Circ 2018;27:484-8.

19. Smith HS. Enteral controlled-release opioid delivery systems. Pain Med 2009;10(Suppl 1):S30-8.

20. Cone EJ. Ephemeral profiles of prescription drug and formulation tampering: evolving pseudoscience on the Internet. Drug Alcohol Depend 2006;83(Suppl 1):S31-9.

21. Tan C, Hansen M, Cohen G, et al. Accuracy of administrative data for identification of patients with infective endocarditis. Int J Cardiol 2016;224:162-4.

22. Addressing prescription drug abuse in the United States: current activities and future opportunities. Atlanta: US Centers for Disease Control and Prevention; 2013. Available: www.cdc.gov/drugoverdose/pdf/hhs_prescription_drug_ abuse_report_09.2013.pdf (accessed 2018 Nov. 22).

23. Wisniewski AM, Purdy $\mathrm{CH}$, Blondell RD. The epidemiologic association between opioid prescribing, non-medical use, and emergency department visits. JAddict Dis 2008;27:1-11.

24. Baldwin N, Gray R, Goel A, et al. Fentanyl and heroin contained in seized illicit drugs and overdose-related deaths in British Columbia, Canada: an observational analysis. Drug Alcohol Depend 2018;185:322-7.

25. Wilson W, Taubert KA, Gewitz M, et al. Prevention of infective endocarditis: guidelines from the American Heart Association: a guideline from the American Heart Association Rheumatic Fever, Endocarditis, and Kawasaki Disease Committee, Council on Cardiovascular Disease in the Young, and the Council on Clinical Cardiology, Council on Cardiovascular Surgery and Anesthesia, and the Quality of Care and Outcomes Research Interdisciplinary Working Group. Circulation 2007;116:1736-54.

\section{Competing interests: None declared.}

This article has been peer reviewed.

Affiliations: ICES (Weir, Slater, Jandoc, Garg); Departments of Epidemiology and Biostatistics (Weir, Garg) and Family Medicine (Koivu), and Divisions of Nephrology (Weir, Garg) and Infectious Diseases (Silverman), Department of Medicine, Western University, London, Ont.

Contributors: Matthew Weir, Sharon Koivu and Michael Silverman contributed to the conception of the study, and all authors contributed to the design. Matthew Weir contributed to the acquisition of data, and Justin Slater, Racquel Jandoc and Amit Garg contributed to the acquisition and analysis of data. Matthew Weir, Sharon Koivu and Michael Silverman contributed to the interpretation of results.
Matthew Weir drafted the manuscript, which all of the authors revised. All authors gave final approval of the version to be published and agreed to be accountable for all aspects of the work.

Acknowledgement: The authors thank Eric McArthur for his assistance with statistical analysis and revision of the manuscript. The authors thank IMS Brogan for use of its Drug Information Database.

Disclaimer: This study was supported by ICES Western, which is funded by an annual grant from the Ontario Ministry of Health and LongTerm Care (MOHLTC). Core funding for ICES Western is provided by the Academic Medical Organization of Southwestern Ontario (AMOSO), the Schulich School of Medicine and
Dentistry (SSMD), Western University, and the Lawson Health Research Institute (LHRI). The opinions, results and conclusions reported in this paper are those of the authors and are independent from the funding sources. No endorsement by ICES, AMOSO, SSMD, LHRI or the Ontario MOHLTC is intended or should be inferred. Parts of this material are based on data and information compiled and provided by the Canadian Institute for Health Information (CIHI). However, the analyses, conclusions, opinions and statements expressed herein are those of the authors, and not necessarily those of $\mathrm{CIHI}$.

Accepted: Dec. 11, 2018

Correspondence to: Matthew Weir, matthew. weir@lhsc.on.ca 